\begin{tabular}{|c|c|c|}
\hline & Int.J.Curr.Microbiol.App.Sci (2016) 5(8): 663-668 & \\
\hline & International Journal of Current Microbiology and Applied Sciences & \\
\hline & ISSN: 2319-7706 Volume 5 Number 8 (2016) pp. 663-668 & \\
\hline $\begin{array}{l}\text { EXCELLENT } \\
\text { PUBLISHERS }\end{array}$ & & wwwhijemas,com \\
\hline
\end{tabular}

Original Research Article

http://dx.doi.org/10.20546/ijcmas.2016.508.075

\title{
Prevalence and Detection of Malaria at a Tertiary Care Hospital in Southern Rajasthan, India
}

\author{
Sanjeev Kumar* and Nasir khan \\ Department of Microbiology, PIMS, Udaipur, Rajasthan, India \\ *Corresponding author
}

\section{A B S T R A C T}

Keywords

Rapid card test, Prevalence, Malaria, Age, HRP-2, pLDH.

\section{Article Info}

Accepted:

25 July 2016

Available Online:

10 August 2016
Malaria presents a diagnostic challenge to laboratories in most countries. The majority of malaria cases are found in countries where cost-effectiveness is an important factor and ease of diagnostic test performance and training of personnel are also major considerations. Most new technology for malaria diagnosis incorporates immunochromatographic capture procedures, with conjugated monoclonal antibodies providing the indicator of infection. Preferred targeted antigens are those which are abundant in all asexual and sexual stages of the parasite; currently interest is focused on the detection of histidine-rich protein 2 (HRP-2) from Plasmodium falciparum and parasite specific lactate dehydrogenase (pLDH). The total 1440 blood samples of suspected malaria cases were tested by rapid card method during the period of April 2015 to September 2015. Out of 1440 suspected cases, $100(14.4 \%)$ cases were positive for malaria. The male to female ratio was 2:1. Positive for Plasmodium vivax were (69\%) and Plasmodium falciparum (31\%). The infection is more common in the age group of $>18$ years. Rapid diagnostic tests offer the possibility of more rapid, non-microscopic method for malaria diagnosis. These tests are easy to perform and require little training to interpret the results as comparing with routine microscopic (gold standard) examination, which required time and need a technical expertise.

\section{Introduction}

Malaria is transmitted to humans by mosquitoes of the genus Anopheles. Malaria is known to be caused by four plasmodia species, namely Plasmodium falciparum, Plasmodium vivax, Plasmodium ovale and Plasmodium malariae, with $P$. falciparum being the most lethal. There are increasing reports of a fifth human-infecting species, Plasmodium knowlesi, which has been described in south-east Asian countries
(Amexo et al., 2004; Beadle et al., 1994; Bell et al., 2005; Chin et al., 1968). In addition, of the five species that infect humans, $P$. vivax and $P$. falciparum cause $95 \%$ of infections. P.vivax may be responsible for $80 \%$ of the infections, because this species has the widest distribution in the tropics, subtropics and temperate zones. $P$. falciparum is generally confined to the tropics, $P$. malariae is sporadically distributed and $P$. ovale is 
confined mainly to central West Africa and some South Pacific islands. The fifth human malaria, $P$. knowlesi, a malaria parasite of long-tailed macaque monkeys, has been confirmed in human cases from Malaysian Borneo, Thailand, Myanmar and the Philippines (Dutta et al., 1999). Diagnosis is complicated by $P$. knowlesi and $P$. malariaehaving similar morphology, and it is difficult to differentiate $P$. falciparum, $P$. malariae and $P$. knowlesi by microscopy (Jivabhai et al., 2014; Karlekar et al., 2012). For efficient treatment and management of malaria, rapid and accurate diagnostic testing is needed. Microscopy has been in use for over 100 years and is inexpensive, rapid and relatively sensitive when used appropriately. Microscopy is regarded as the 'gold standard' for malaria diagnosis (Kilian et al., 2000). However, the lack of skilled technologists in medical facilities in affected areas often leads to poor interpretation of data. Furthermore, microscopy is time consuming and labour intensive, cannot detect sequestered $\mathrm{P}$. falciparum parasites and is less reliable at low-density parasitaemia $(<50$ parasites $/ \mathrm{ml}$ blood) (Lee et al., 2009; Lee et al., 2011b; Leke et al., 1999).

Most of the point prevalence studies in India have been carried out for outbreak/epidemic investigations. There is very limited information on age and sex specific seasonal prevalence of malaria in different paradigms in the country. The burden is generally higher in men than women in all age groups. Children in the states of Assam (Ong et al., 2009), Arunachal Pradesh (Palmer et al., 1998), and Rajasthan (Patriia) had a higher incidence of malaria than adults. Malaria is considered to be immediately life threating and a patient with the diagnosis of $P$. falciparum or $P$. knowlesi malaria should be considered a medical emergency because the disease can be rapidly fatal.
Clinical misdiagnosis of malaria has been reported both in the public and private health sectors. Both under and over diagnosis of malaria has been observed in South East Asian countries (Amexo et al., 2004). Hence it is necessary to use appropriate diagnostic tools to prevent unethical use of antimalarials in the malaria negative patients (WHO, 2006). The development and commercial availability of Rapid diagnostic tests either for individual species (for P. falciparum) or in combination (P. falciparum $+\mathrm{P}$. vivax) or for all the species has revolutionized malaria diagnosis recently (Palmer et al., 1998; Valecha et al., 2003). The standard test particularly HRP-2 based for P. falciparum has acceptably high sensitivity and specificity (Beadle et al., 1994). With the reducing cost of these tests, RDTs are now being increasingly deployed in the control programs in India. The major advantage is that RDT can be used for onthe-spot diagnosis and treatment of malaria and in many situations could be lifesaving as well as a useful tool for transmission control at the community level.

Although RDT's for malaria diagnosis have been introduced in the National Policy, there use should be further encouraged as a part of the EDPT policy being followed in India. Besides routine diagnosis during both active and passive case detection, they can also play an important role in the special situations e.g. in spot detection and treatment of cases in migrant population/refugees, in large projects where labor congregates and during outbreaks of malaria.Our aim of the study is to detect malarial parasitic infections among patients attending our tertiary care hospital and to know the prevalence.

\section{Materials and Methods}

This study was carried out at the department of Microbiology, central clinical laboratory, 
Pacific institute of Medical Sciences, Udaipur, Rajasthan, over a period of April 2015 to September 2015.

Study type: Retrospective type of study.

\section{Sample collection}

The patient's name, age, sex, details of history and clinical examination findings were recorded. After obtaining informed consent, $2 \mathrm{ml}$ of blood collected in EDTA (anticoagulant) containing vial from antecubital vein of all patients by taking sterile precautions.

\section{Rapid diagnostic test (RDT)}

Malaria antigens suitable for rapid diagnostic tests are HRP-2 and $\mathrm{pLDH}$. The detection system for P.falciparum malaria is based on the detection of P.falciparum specific histidine rich protein-2 (Pf.HRP-2), which is a water soluble protein that is released from parasitized erythrocytes of infected individuals. The detection system for P.vivax malaria is based on presence of P.vivax specific $\mathrm{pLDH}$.

Malaria Antigen utilizes the principle of immunochromatography. As the test sample flows through the membrane assembly of the device after addition of the clearing buffer, the colored colloidal gold conjugates of monoclonal anti-Pf. HRP-2 (IgG) antibody and monoclonal anti-P.vivax specific pLDH antibody complexes the HRP-2/pLDH in the lysed sample. This complex moves further on the membrane to the test region where it is immobilized by the anti-Pan specific pLDH (monoclonal) antibody and/or the monoclonal antiPf.HRP-2(IgM) antibody coated on the membrane leading to formation of pinkpurple colored band/s which confirms a positive result. A band will appear under Pf at the test region in falciparum positive samples, while a band will appear under $\mathrm{Pv}$ in vivax malaria positive samples. Appearance of band under Pf as well as $\mathrm{Pv}$ in the test region suggests a mixed infection.

\section{Results and Discussion}

Total 1440 suspected cases were studied during the period of six months, out of which 100 cases were positive for malaria. The prevalence rate was $14.4 \%$. Prevalence of malaria was more in males (63\%) as compared to females (37\%)[Graph 1].

Among the 100 cases of malaria positive cases, Plasmodium vivax was predominant (69\%) and Plasmodium falciparum was $(31 \%)$. In this study malaria infection was more common in the age group of $>18$ years $(71 \%)$, as compared to $\leq 18$ years $(29 \%)$ [Table 1].

The present study detected all the positive malaria cases by rapid diagnostic method (Immuno-chromatography method). Immuno-chromatographic technique offers the possibility of more rapid, nonmicroscopic methods for malaria diagnosis, thereby saving on training and time. These tests are easy to perform and require little training to interpret the results.

However, microscopy is regarded as the 'gold standard' for malaria diagnosis (WHO, 1999). However, the lack of skilled technologists in medical facilities in affected areas often leads to poor interpretation of data. Furthermore, microscopy is time consuming and labour intensive, cannot detect sequestered P. falciparum parasites (Leke et al.,., 1999) and is less reliable at low-density parasitaemia $[<50$ parasites $(\mathrm{ml}$ blood) (Kilian et al., 2000; Bell et al., 2005)]. None the less, a good microscopist can differentiate species with microscopy. The prevalence of malaria infection in our study was found to be (14.4\%). Male to 
female ratio was $2: 1$. Species distribution was P.vivax (69\%) and P.falciparum (31\%). Maximum prevalence was found to be in the month of July and Agust. Malaria infection detected in all age groups with maximum prevalence in age group $>18$ years.

There is a wide variation of reports of prevalence of malarial infection in India and other countries. This can be due to differences in geographical and climatic condition which affect mosquito breeding, socio-economic conditions of patients, knowledge about healthcare and public health practices.

Prevalence of malarial infection in our study was $14.4 \%$ which is similar to Singh et al., of Navi Mumbai(16.58\%),Pandey et al., of Bilaspur (24.74\%). However Hadiyaet al., from Gujarat and Karlekar et al., from Gadchiroli (Maharashtra) reported very less prevalence of $2.10 \%$ and $4.28 \%$ respectively. This difference could be due to geographical and climate conditions.

However, our study period is only 6 months (April to September), maximum numbers of cases were found in the months of July and August. Similar findings are reported by Singh et al., Sachin et al., Hadiya et al., The high prevalence of malaria in this period could be due to collection of water in rainy season and mosquito breeding.

Regarding prevalence of species, Plasmodium vivax was $69 \%$, Plasmodium falciparum $31 \%$. Our findings are similar to Hadiyaet al., who reported $P$. vivax $61.41 \%$ $P$. falciparum $38.56 \%$, but different from Karlekar et al., who reported Plasmodium vivax $33.8 \%$ and Plasmodium falciparum $66.6 \%$. The difference in prevalence of Plasmodium vivax and Plasmodium falciparum in different areas can be due to presence of endemicity of particular type and higher relapses in vivax type.

Table.1 Detection of Malaria antigens by rapid card method and distribution among male and females

\begin{tabular}{|c|c|c|c|c|c|}
\hline \multirow{2}{*}{$\begin{array}{c}\text { Species of } \\
\text { Malaria } \\
\text { parasites }\end{array}$} & \multicolumn{2}{|c|}{ Children $(\leq 18$ years $)$} & \multicolumn{2}{|c|}{ Adults $(>18$ years $)$} & \multirow{2}{*}{ Total } \\
\cline { 2 - 5 } & Male & Female & Male & Female & \\
\hline$P$. falciparum & 07 & 05 & 12 & 07 & 31 \\
\hline$P$. vivax & 10 & 07 & 34 & 18 & 69 \\
\hline Total & 17 & 12 & 46 & 25 & 100 \\
\hline
\end{tabular}

Graph.1 Gender wise distribution of Malaria infection

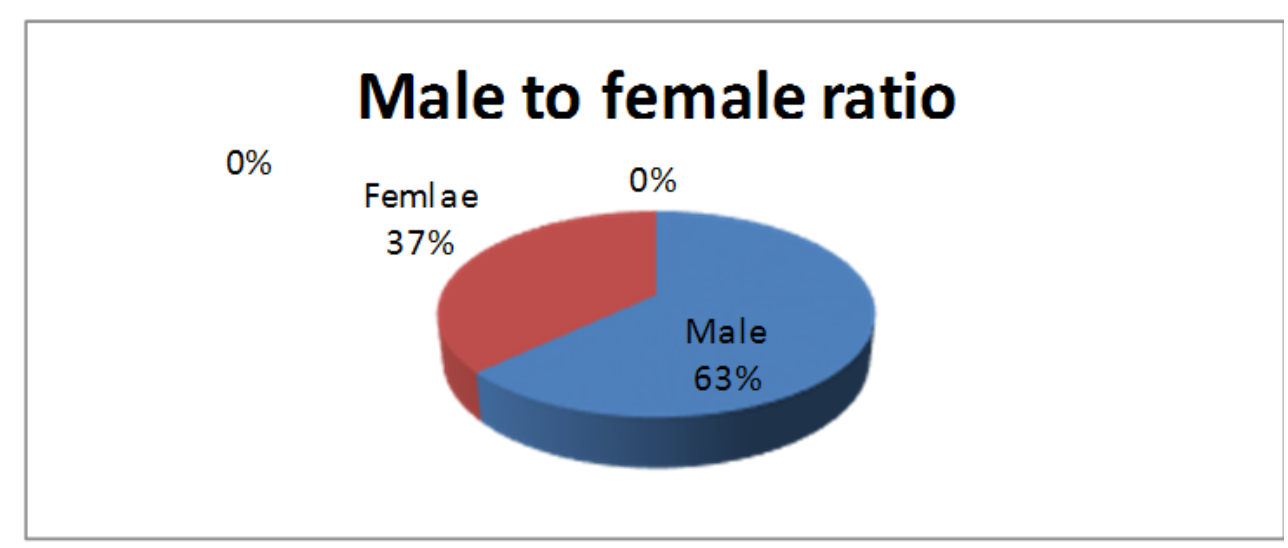


Male to female ratio in our study was $2: 1$, which is similar to Karlekar et al., and Singh et al., reports. The difference in $\mathrm{M}: \mathrm{F}$ ratio could be due to various reasons like body odour, which may attract mosquitoes, movement of males in wider areas, more chances of mosquito bites and some unknown inherent susceptibility. In our study the maximum number of cases of malaria occurred in the age group $>18$ years (63\%). Our finding correlates with S.R. Karlekar et al., who reported mean age group of 24.8 years and Singh et al., reported 21-30 years of age. The reason of higher prevalence in this age group could be due to movement in wider areas possibly endemic, more chances of exposure to mosquito bites.

In conclusion, parasitological confirmation of suspected malaria using microscopy, the gold standard, is cumbersome and requires trained personnel, microscopes and a source of electricity. PfHRP2- and pLDH-based RDTs are the most commonly used. These tests are easy to perform and require little training to interpret the results. Therefore, malaria treatment based on RDTs, are quick in endemic and remote areas, where limited sources.

The present study reveals a prevalence rate of malarial infection (14.4\%) in tertiary care hospital, Udaipur (India). Malarial infections were more in males than females and infection occurred in age group $>18$ years. This finding could be due to more chances of exposure of mosquito bites in endemic areas.

\section{References}

Amexo, M., Tolhurst, R., Barnish, G. and Bates, I. 2004. Malaria Misdiagnosis: effects on the poor and vulnerable; Lancet, 364: 1896-1898.
Beadle, C., Long, G.W., Weiss, W.R., McElroy, P.D., Maret, S.M., Oloo, A.J. and Hoffman, S.L. 1994. Diagnosis of malaria by detection of Plasmodium falciparum HRP-2 antigen with a rapid dipstick antigen-capture assay; Lancet, 343: 564-568.

Bell, D.R., Wilson, D.W. \& Martin, L.B. 2005. False-positive results of a Plasmodium falciparum histidine-rich protein 2-detecting malaria rapid diagnostic test due to high sensitivity in a community with fluctuating low parasite density. Am. J. Trop. Med. Hyg., 73: 199- 203.

Chin, W., Contacos, P. G., Collins, W. E., Jeter, M. H. \& Alpert, E. 1968. Experimental mosquito-transmission of Plasmodium knowlesi to man and monkey. Am. J. Trop. Med. Hyg., 17: 355-358.

Dutta, P., Khan, A.M., Mahanta, J. 1999. Problem of malaria in relation to Sociocultural diversity in some ethnic communities of Assam and Arunachal Pradesh. J. Parasitic Dis., 23: 101-104.

Jivabhai, H.T., Kanubhai, V.B., Hariharbhai, A.A., Animeshbhai, D.G., Kanubhai, V.V. 2014. Retrospective study of malaria cases attending at tertiary care level hospital in Rajkot city, Gujarat. Int. J. Curr. Res., 6: 6273-6276.

Karlekar, .S.R., Deshpande, M.M., Andrew, R.J. 2012. Prevalence of Asymptomatic Plasmodium vivax and Plasmodium falciparum Infections in Tribal Population of a Village in Gadchiroli District of Maharashtra State, India. Biol. Forum-An Int. J., 4: 42-44.

Kilian, A.H., Metzger, W.G., Mutschelknauss, E.J., Kabagambe, G., Langi, P., Korte, R. \& von Sonnenburg, F. 2000. Reliability of malaria microscopy in epidemiological studies: results of quality control. Trop. Med. Int. Health, 5: 3-8.

Lee, K.S., Cox-Singh, J. \& Singh, B. 2009. Morphological features and differential counts of Plasmodium knowlesi 
parasites in naturally acquired human infections. Malar J., 8: 73.

Lee, K.S., Divis, P.C.S., Zakaria, S.K., Matusop, A., Julin, R. A., Conway, D. J., Cox-Singh, J. \& Singh, B. 2011 b. Plasmodium knowlesi: reservoir hosts and tracking the emergence in humans and macaques. PloS Pathog., 7: e1002015.

Leke, R.F.G., Djokam, R.R., Mbu, R., Leke, R.J., Fogako, J., Megnekou, R., Metenou, S., Sama, G., Zhou, Y. \& other authors .1999. Detection of the Plasmodium falciparum antigen histidine- rich protein 2 in blood of pregnant women: implications for diagnosing placental malaria. J. Clin. Microbiol., 37: 2992-2996.

Ong, C.W., Lee, S.Y., Koh, W.H., Ooi, E. E. \& Tambyah, P.A. 2009. Monkey malaria in humans: a diagnostic dilemma with conflicting laboratory data. Am. J. Trop. Med. Hyg., 80: 927928.

Palmer, C.J., Lindo, J.F., Kaminsky. R.Q., Bamm, M.K. and Ager, A.L. 1998. Evaluation of the OptiMAL test for rapid diagnosis of Plasmodium vivax and Plasmodium falciparum malaria; $J$. Clinic. Microbiol., 36: 203-206.

Patriia, M. Tille, Bailey \& Scott's. Diagnostic Microbiology, $13^{\text {th }}$ Edn, Chapter 49;pg 624.

Prakash, A., Mohapatra, P.K., Bhattacharyya, D.R., Doloi, P., Ma- hanta, J. 1997. Changing malaria endemicity-a village based study in Sonitpur, Assam. J. Com. Dis., 29: 175-178.

Sachin, P., Arun, S. 2012. A retrospective study of characteristics of Malaria cases attending OPD of a tertiary care level hospital in Bilaspur district, Chhattisgarh. National J. Community Med., 3: 218-220.

Shukla, R.P., Pandey, A.C., Mathur, A. 1995. Investigations of malaria outbreak in Rajasthan. Indian J. Malariol., 32: 119128.

Singh, G., Urhekar, A.D., Maheshwari, U., Sharma, S., Raksha. 2005. Prevalence of Malaria in a Tertiary hospital in Navi Mumbai, India. J. Bacteriol. Parasitol., 6(2):221-224.

Singh, B., Daneshvar, C. 2010. Plasmodium knowlesi malaria in Malaysia. Med. J. Malaysia, 65: 166-172.

Valecha, N., Singh, N., Yadav, R.S., Dev, V., Aggarwal, A. and Subbarao, S.K. 2003. Field evaluation of OptiMAL rapid malaria diagnostic test in India; ActaParasitol, 48: 229-232.

Van den Eede, P., Van, H. N., Van Overmeir, C., Vythilingam, I., Duc, T. N., Hung le, X., Manh, H. N., Anne ', J., D'Alessandro, U. \&Erhart, A. 2009. Human Plasmodium knowlesi infections in young children in central Vietnam. Malar J. 8: 249.

WHO. 1999. New Perspectives: Malaria Diagnosis. Report of joint WHO/USAID informal consultation 2527 October 1999. Report No. WHO/CDS/RBM/2000.14, WHO/MAL/2000.1091. Geneva: World Health Organization. http://wholibdoc. who.int/hq/2000/WHO__CDS_RBM_ 2000.14.pdf.

World Health Organization. 2006. Guidelines for the treatment of malaria; WHO/HTM/MAL/2006.1108; www.searo.who.int; www.nvbdep.gov.in.

\section{How to cite this article:}

Sanjeev Kumar and Naseer Khan. 2016. Prevalence and Detection of Malaria at a Tertiary Care Hospital in Southern Rajasthan, India. Int.J.Curr.Microbiol.App.Sci. 5(8): 663-668. doi: http://dx.doi.org/10.20546/ijcmas.2016.508.075 